\title{
Rydberg Atoms in "Circular" States
}

\author{
Randall G. Hulet and Daniel Kleppner \\ Research Laboratory of Electronics and Department of Physics, Massachusetts Institute of Technology, \\ Cambridge, Massachusetts 02139
}

(Received 5 August 1983)

\begin{abstract}
The population of a beam of lithium atoms has been transferred from a low angular momentum Rydberg state to a state with $|m|=n-1$, with nearly $100 \%$ efficiency. Selective field ionization is employed to detect the high $|\mathrm{m}|$ state. Transfer occurs by a series of adiabatic rapid passages; it can also occur by a single multiphoton process.
\end{abstract}

PACS numbers: $32.80 . \mathrm{Kf}, 32.90 .+\mathrm{a}$

We report the production of a population of atoms in a Rydberg state with $|m|=n-1$, where $m$ and $n$ are the magnetic and principal quantum numbers, respectively. We designate these states as "circular" because in the classical limit they describe an electron in a circular orbit. Among all the states with a given principal quantum number, the circular states have the largest magnetic moment, smallest Stark effect, and longest radiative lifetime. Atoms in circular states are expected to exhibit highly anisotropic collision cross sections. Only one electric dipole transition is available $(n-n-1 ;|m|-|m|-1)$, allowing circular states to serve as useful approximations to two-level systems for studies of the electrodynamics of atoms in cavities. In addition, the circular states are well suited to very high precision Rydberg-state spectroscopy.

Research with circular states has not been possible because of the lack of an experimental technique for populating them. The usual laser techniques for exciting Rydberg atoms cannot be used to populate a circular state since each photon carries only a single unit of angular momentum. Freeman and Kleppner ${ }^{1}$ have proposed a "Stark switching" method to transfer atoms to high angular momentum states, but this produces high-l rather than high- $m$ atoms. Koch and Mariani ${ }^{2}$ have used laser excitation of collisionally excited Rydberg atoms to selectively populate Rydberg states, though the method has not yet been applied to the production of atoms in high- $m$ states. A method involving optical excitation of microwave "dressed states" has been proposed by Stroud and Molander. ${ }^{3}$ Simple microwave excitation can spread the population among many levels, but cannot transfer it uniquely to the circular state.

We have developed a simple method for transferring a population of atoms in a low- $m$ Rydberg state to a circular state with essentially $100 \%$ efficiency. The atoms are transferred by a series of adiabatic rapid passage $\mathrm{s}^{4}$ induced by a microwave field and an electric field which changes linearly with time. Each adiabatic rapid passage causes $|m|$ to increase by 1 ; the process terminates when the atom reaches the circular state. We have demonstrated the method with lithium, $n=19$, but to explain the operating principle we shall consider hydrogen, $n=4$, neglecting electron and nuclear spin.

The energy of hydrogen (in inverse centimeters) in an electric field $F$ is given to first order by $^{5} W=-\left[1 / n^{2}-3 n F\left(n_{1}-n_{2}\right)\right] R$, where $n_{1}$ and $n_{2}$ are the parabolic quantum numbers $\left(n_{1}+n_{2}+|m|\right.$ $+1=n), R$ is the Rydberg constant, and the field $F$ is in atomic units, $5.14 \times 10^{9} \mathrm{~V} \mathrm{~cm}^{-1}$. Energy levels for $n=4$ are shown in Fig. 1(a). The lowest-lying $|m|=0$ state $\left(n_{1}=0\right)$ is initially populated by laser excitation. A series of transitions satisfying $\Delta|m|=1, \Delta n_{1}=0$ is induced by adiabatic rapid passage, as indicated by the heavy arrows. Because of the second-order Stark effect [not shown in Fig. 1(a)], the transitions occur consecutively in time. As will be explained, the energies evolve so that $\Delta|m|=-1$ transitions cannot occur. Consequently, the entire population is transferred to the circular state.

To further illustrate the process, the energy levels for the $n_{1}=0$ states are shown in Fig. 1(b) with the second-order Stark effects $W_{2}$ included and exaggerated for clarity. The microwave frequency is slightly below the resonance frequency for the initial transition $|m|=0 \rightarrow 1$. As the field decreases, the transitions are consecutively encountered as shown.

The resonance condition for the transitions shown in Fig. 1 can be written $\nu=\nu_{1}+\nu_{2}$, where $\nu_{1}=(3 n F) c R$ is the first-order transition frequency, and $\nu_{2}$ is a second-order correction: $\nu_{2}$ $=3 n^{4} F^{2}(|m|-C) c R$, where $C=(n-3) / 4$. For the "leakage" transitions $\left(\Delta|m|=-1, \Delta n_{1}=+1\right)$ shown as dashed arrows in Fig. 1(a), $\nu_{2}{ }^{\prime}=-\frac{3}{2} n^{4} F^{2}$ $\times(|m|+2 C) c R$. Because of the negative sign of 
(a)

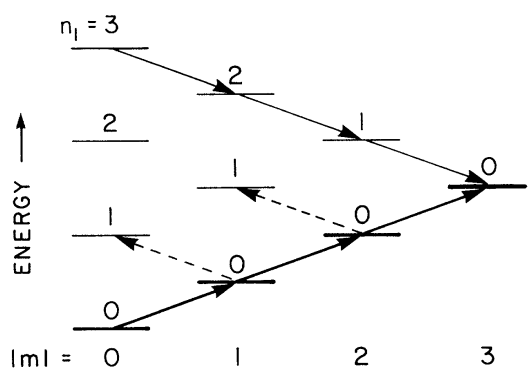

(b)

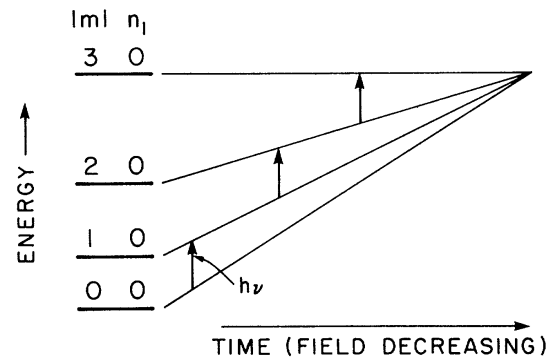

FIG. 1. Schematic diagram of the excitation process, illustrated with hydrogen, $n=4$. (a) Energy levels in an electric field, neglecting the second-order Stark effect. The bold arrows show the excitation path used to populate the circular state, $|m|=3$; the light arrows show an alternative excitation route; the dashed arrows show "leakage" transitions which must be avoided. (b) The progression of the $n_{1}=0$ levels in a decreasing field, with the second-order Stark effect exaggerated for clarity. An adiabatic rapid transition can occur whenever the energy level separation passes through resonance with the microwave frequency $\nu$. Because of the second-order Stark effect these transitions occur successively, "stepping" the population along the route shown in (a).

$\nu_{2}{ }^{\prime}$, these transitions are not encountered as the field decreases.

The experiment employed the $n=19$ manifold of lithium. General methods for exciting and detecting Rydberg atoms are described in Ref. 5. The field was approximately $830 \mathrm{~V} \mathrm{~cm}^{-1}$ and the frequency $\nu$ was $30 \mathrm{GHz}$. Because of core perturbations which drastically change the resonance frequency for the $|m|=0 \rightarrow 1$ and $1 \rightarrow 2$ transitions, the initial state was chosen to be $|m|=2$. An atomic beam was used and the Rydberg state was populated by two-step pulsed excitation: $2^{2} S_{1 / 2}$ $\rightarrow 2^{2} P_{3 / 2}(671 \mathrm{~nm})$ and $2^{2} P_{3 / 2} \rightarrow$ Rydberg state $(\sim 354$ $\mathrm{nm})$. A dye laser excited the first step; a frequency-doubled dye laser excited the second. An electric field was applied with use of copper field plates which were carefully spaced $6.9 \mathrm{~mm}$ apart. Ions were collected through a grid drilled in the lower plate over a length of $20 \mathrm{~mm}$. The interaction region was irradiated by $\sim 10^{-4} \mathrm{~W}$ of micro- wave power at $30 \mathrm{GHz}$ from a $8 \times 80-\mathrm{mm}^{2}$ horn, with the $E$ field parallel to the field plates. Because the laser light was linearly polarized, both $n=19, n_{1}=0, m=|2|$ states were populated. And since the microwave field was linearly poarized in the same direction, both of the circular states $(m= \pm 18)$ were populated, as well.

The sequence of events is as follows: The atoms are excited by 5-ns laser pulses in the 830$\mathrm{V} \mathrm{cm}{ }^{-1}$ field. The microwave power is switched on and the field is linearly decreased to $806 \mathrm{~V}$ $\mathrm{cm}^{-1}$ during a $5-\mu \mathrm{s}$ period. The first transition $(|m|=2 \rightarrow 3)$ occurs at $824 \mathrm{~V} \mathrm{~cm}^{-1}$; the last $(|m|$ $=17 \rightarrow 18$ ) occurs at $810 \mathrm{~V} \mathrm{~cm}^{-1}$. The microwave power is switched off and the population is analyzed by selective field ioization ${ }^{5}$ : The field is abruptly switched to $4.4 \mathrm{kV} \mathrm{cm}^{-1}$ and then ramped to $6.0 \mathrm{kV} \mathrm{cm} \mathrm{cm}^{-1}$ in a $2-\mu \mathrm{s}$ period. The strength of the field required to ionize $n_{1}=0$ states increases with $|m|$. Thus, the later the ionization peak, the higher the value of $|m|$. The ionization signal is recorded by a transient analyzer.

Experimental results are shown in Fig. 2. These curves represent an average of about 100 laser pulses. Approximately fifty atoms are excited in each pulse. To demonstrate the progression of the population transfer, the "on" time, $\tau_{\mathrm{rp}}$, of the rapid-passage field ramp and of the microwave power was successively increased. In curve $a, \tau_{\mathrm{rp}}<1 \mu \mathrm{s}$; no transitions have occurred and the initial states, $n=19,|m|=2$, are displayed. [Because of the finite laser linewidth $\left(0.1 \mathrm{~cm}^{-1}\right)$ and power broadening effects, a smaller number of $m=0$ atoms are also excited. Their ionization signal can be discerned just before the $|m|=2$ signal.]

As the time $\tau_{\mathrm{rp}}$ is increased the ionization signal occurs progressively later, indicating that the atoms are being transferred to higher $|m|$ states. Ionization signals from adjacent $|m|$ states overlap in this region, but as higher values of $|m|$ are reached the signals start to be resolved. The signal in curve $b$ is predominantly due to $|m|=17$, while $c$ shows contributions from $|m|=17$ and 18. Finally, for $\tau_{\mathrm{rp}}>4.5 \mu \mathrm{s}$, the circular state, $|m|=18$, is populated, as shown in curve $d$. No further changes occur with increasing $\tau_{\mathrm{rp}}$. The shape and position of the signals agree with ionization rate calculations for hydrogen based on the results of Damburg and Kolosov ${ }^{6}$ to within our ability to measure the ionizing field and the field's rate of change. The conspicuous difference in shape between the $|m|$ $=2$ signal and the high $-|m|$ signals is due pre- 


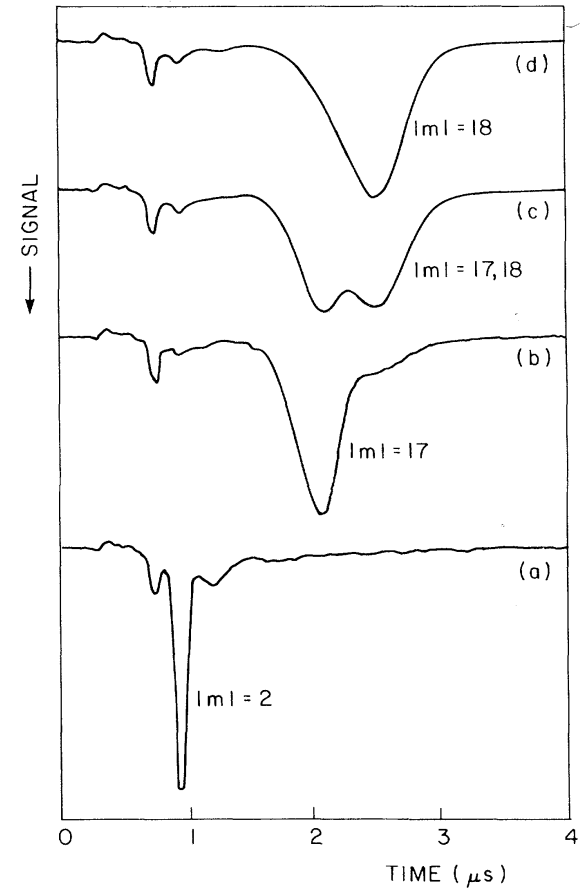

FIG. 2. Distribution of population in lithium for various values of $|m|$ as revealed by selective field ionization. States are $n=19, n_{1}=0$. The ionization field increases with time. The ionization thresholds occur in increasing fields as $|m|$ increases. Curve $a:|m|=2$ states initially populated by laser excitation in a field of $830 \mathrm{~V} \mathrm{~cm}^{-1}$. The signal is clipped because of saturation of the detector. The small peak to the left is due to $|m|=2$ atoms which ionize hydrogenically. The $|m|$ $=2$ peaks occurs at approximately $4.5 \mathrm{kV} \mathrm{cm}^{-1}$ (the actual ionization field is somewhat less, since the low$m$ states ionize during the rapidly rising portion of the pulse and there is a delay in detection). Curve $b$ : Same as $a$, but with the adiabatic rapid-passage field ramp on for a time $\tau_{\text {rp }}=4 \mu \mathrm{s}$. The $|m|=2$ population has been transferred predominantly to $|m|=17$. Curve $c: \tau_{\text {rp }}$ increased; ionization signals for $|m|=17$ and 18 are both visible. Curve $d: \tau_{\mathrm{rp}}>4.5 \mu \mathrm{s}$. The $|m|=18$ circular states is populated. No further change in the ionization signal occurs with increasing $\tau_{\mathrm{rp}}$. The ionizing field has approximately the following values: $0 \mu \mathrm{s}, 820 \mathrm{~V} \mathrm{~cm}^{-1} ; 1 \mu \mathrm{s}, 4.6 \mathrm{kV} \mathrm{cm}^{-1} ; 2 \mu \mathrm{s}, 5.7 \mathrm{kV} \mathrm{cm}^{-1}$; $3 \mu \mathrm{s}, 6.0 \mathrm{kV} \mathrm{cm}^{-1} ; 4 \mu \mathrm{s}, 6.1 \mathrm{kV} \mathrm{cm}^{-1}$.

dominantly to nonhydrogenic effects in the $|m|=2$ manifold. ${ }^{5}$

We have also populated the circular state via the route shown by the light arrows in Fig. 1(a). This route requires use of an increasing field. The frequency was again $30 \mathrm{GHz}$, and the field was slewed from 814 to $835 \mathrm{~V} \mathrm{~cm}^{-1}$ in $5 \mu \mathrm{s}$. Along this route, the ionization signal occurs at successively lower fields as $|m|$ increases, the final signal coinciding with curve $d$ in Fig. 2.

The experiment was planned so that the successive transitions would be well isolated, that is, under conditions in which only two levels at a time are significantly populated. In this case the probability for successfully undergoing an adiabatic transition from $\left|m_{j}\right|$ to $\left|m_{j}\right|+1$ is $^{4}$

$$
P_{j}=1-\exp \left[-\frac{1}{2} \pi\left|V_{j}\right|^{2} / h\left(d W_{j} / d t\right)\right] \text {. }
$$

Here $V_{j}$ is the matrix element of the oscillating field. (In the dressed-atom picture, $V_{j}$ is the level separation at the avoided crossing.) $W_{j}$ is the energy difference between the two levels in the absence of the microwave field. Efficient transfer requires a large value for the matrix element $V_{j}$ and a small value of the traversal rate $d W_{j} / d t$. The maximum value of $V_{j}$ was limited by the available microwave power, approximately 10 $\mathrm{mW}$. The minimum rate of $d W / d t$ was fixed by the available interaction time. We allowed $5 \mu \mathrm{s}$ for the total rapid-passage process. Under our operating conditions, we estimate that $P_{j} \sim 1-10^{-5}$ and that the total loss in population for the sixteen transitions $(|m|=2 \rightarrow 18)$ is approximately $2 \times 10^{-4}$. We saw no evidence for atoms being left stranded in intermediate $|m|$ states, though a small background of residual $|m|=2$ atoms, less than $1 \%$, can be discerned in the data. This may be due to starting the rapid-passage field ramp too close to the first transition.

If the microwave power is increased, interference effects are to be expected as more than two levels become significantly populated at the same time. In the limit of a very strong microwave field, all the states can be simultaneously excited and the process can be viewed as a single multiphoton event. We have investigated how the states evolve by numerically solving Schrödinger's equation for the four-level sy stem shown in Fig. 1 (b) for various slew rates and microwave fields. Results for well separated transitions are shown in Fig. 3(a). The population progressively "jumps" to states of increasing $|\mathrm{m}|$. In contrast, in a high microwave field the system evolves through a superposition of all the states, as shown in Fig. 3 (b). Even in this case, however, none of the intermediate $|\mathrm{m}|$ states remains populated; the transfer to the circular state appears to be $100 \%$ efficient. Thus, in principle the circular state can be excited by a single multiphoton step. In practice, if the microwave field strength is increased sufficiently, "leakage transitions" $(\Delta|m|$ $=-1$ ) are expected to occur. With the microwave field and slew rate used in our experiment, two 

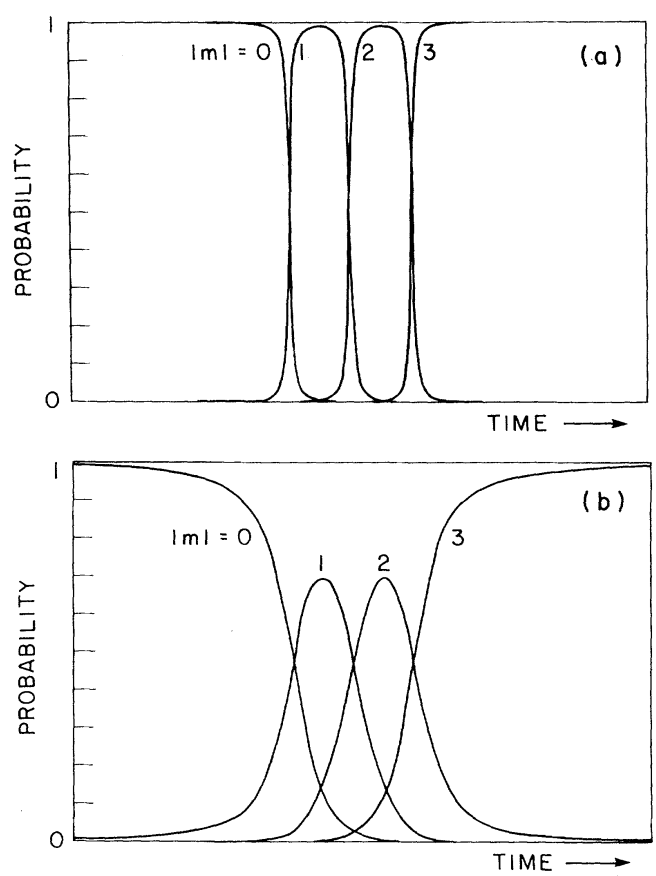

FIG. 3. (a) Results of numerical computation of the evolution of populations for the $n_{1}=0$ states shown in Fig. 1. The population is transferred in a series of well defined steps. (Slew rate, $-1 \mathrm{kV} \mathrm{cm} \mathrm{cm}^{-1} \mathrm{~s}^{-1}$; intensity, $3 \times 10^{-7} \mathrm{~W} \mathrm{~cm}^{-2} ; \nu=30 \mathrm{GHz}$.) (b) Same system but with overlapping transitions. [The time scale is approximately 88 times smaller than that of (a).] The population is transferred by a single multiphoton step. (Slew rate, $-100 \mathrm{kV} \mathrm{cm}{ }^{-1} \mathrm{~s}^{-1}$; intensity, $3 \times 10^{-5} \mathrm{~W}$ $\mathrm{cm}^{-2} ; \nu=30 \mathrm{GHz}$.)

or three intermediate levels can have significant populations at a given time, as in Fig. $3(\mathrm{~b})$.
The production of atoms in circular states opens the way to the study of atom-cavity effects such as inhibited spontaneous emission, ${ }^{7}$ and facilitates the measurement of the Rydberg constant by millimeter-wave spectroscopy. One can also look forward to studies with circular states including magnetic deflection, field ionization, diamagnetic interactions, and collisional effects.

We are grateful to L. Moi, K. E. Plonty, J. -M. Raimond, E. H. Schadler, and W. P. Spencer for assistance. Also, we thank Quantel International for lending us a Model 581-10 yttrium aluminum garnet laser. This work was sponsored by the U. S. Joint Services Electronics Program (Contract No. DAAG29-80-C-0104) the U. S. Office of Naval Research (Grant No. NR393-039), and the National Science Foundation (Grant No. PHY8306273).

${ }^{1}$ R. R. Freeman and D. Kleppner, Phys. Rev. A $\underline{14}$, 1614 (1976).

${ }^{2}$ P. M. Koch and D. R. Mariani, J. Phys. B $\underline{13}$, L645 (1980).

${ }^{3}$ C. R. Stroud, Jr., private communication.

${ }^{4}$ J. R. Rubbmark, M. M. Kash, M. G. Littman, and D。 Kleppner, Phys. Rev. A 23, 3107 (1981).

${ }^{5}$ D. Kleppner, M. G. Littman, and M. L. Zimmerman, Rydberg States of Atoms and Molecules, edited by R. F. Stebbings and F. B. Dunning (Cambridge Univ. Press, Cambridge, England, 1982), p. 73.

${ }^{6}$ R. J. Damburg and V. V. Kolosov, J. Phys. B 12 , 2637 (1979).

${ }^{7}$ D. Kleppner, Phys. Rev. Lett. 47, 233 (1981)。 